\title{
Experimental Demonstration of Fusion-Relevant Conditions in Magnetized Liner Inertial Fusion
}

\author{
M. R. Gomez, S. A. Slutz, A. B. Sefkow, D. B. Sinars, K. D. Hahn, S. B. Hansen, E. C. Harding, P. F. Knapp, \\ P. F. Schmit, C. A. Jennings, T. J. Awe, M. Geissel, D. C. Rovang, G. A. Chandler, G. W. Cooper, M. E. Cuneo, \\ A. J. Harvey-Thompson, M. C. Herrmann, M. H. Hess, O. Johns, D. C. Lamppa, M. R. Martin, R. D. McBride, \\ K. J. Peterson, J. L. Porter, G. K. Robertson, G. A. Rochau, C. L. Ruiz, M. E. Savage, I. C. Smith, \\ W. A. Stygar, and R. A. Vesey \\ Sandia National Laboratories, P.O. Box 5800, Albuquerque, New Mexico 87185, USA
}

(Received 18 June 2014; published 6 October 2014)

\begin{abstract}
This Letter presents results from the first fully integrated experiments testing the magnetized liner inertial fusion concept [S. A. Slutz et al., Phys. Plasmas 17, 056303 (2010)], in which a cylinder of deuterium gas with a preimposed $10 \mathrm{~T}$ axial magnetic field is heated by $Z$ beamlet, a $2.5 \mathrm{~kJ}, 1 \mathrm{TW}$ laser, and magnetically imploded by a 19 MA, 100 ns rise time current on the $Z$ facility. Despite a predicted peak implosion velocity of only $70 \mathrm{~km} / \mathrm{s}$, the fuel reaches a stagnation temperature of approximately $3 \mathrm{keV}$, with $T_{e} \approx T_{i}$, and produces up to $2 \times 10^{12}$ thermonuclear deuterium-deuterium neutrons. X-ray emission indicates a hot fuel region with full width at half maximum ranging from 60 to $120 \mu \mathrm{m}$ over a $6 \mathrm{~mm}$ height and lasting approximately $2 \mathrm{~ns}$. Greater than $10^{10}$ secondary deuterium-tritium neutrons were observed, indicating significant fuel magnetization given that the estimated radial areal density of the plasma is only $2 \mathrm{mg} / \mathrm{cm}^{2}$.

DOI: 10.1103/PhysRevLett.113.155003

PACS numbers: 52.58.Lq
\end{abstract}

Inertial confinement fusion (ICF) concepts rely on implosion velocities greater than $300 \mathrm{~km} / \mathrm{s}$ and spherical convergence of fuel to achieve the high temperatures $(T>4 \mathrm{keV})$ and areal densities $\left(\rho R>0.3 \mathrm{~g} / \mathrm{cm}^{2}\right)$ required for hot spot ignition [1-3]. Magneto-inertial fusion (MIF) concepts attempt to significantly relax implosion velocity and pressure requirements while still achieving high temperatures through the use of insulating magnetic fields, which decrease thermal conductivity losses normal to the field and increase fusion product confinement [4,5]. This Letter discusses the successful demonstration of an MIF concept referred to as magnetized liner inertial fusion (MagLIF) [6,7].

Recently, axial magnetic fields were applied to laserdriven ICF targets at the Omega facility [8,9], where they were found to suppress heat losses by approximately $50 \%$, which increased ion temperatures by $15 \%$ and produced $30 \%$ greater neutron yield. This result demonstrated magnetothermal insulation benefits, but the improvement was limited by the spherical capsule geometry. In MagLIF, the cylindrical implosion is complementary to the axial magnetic field. The large magnetic field and substantial axial fuel extent at stagnation are expected to efficiently trap alpha particles [6]; thus, a greater increase in performance due to the magnetic field is anticipated.

Figure 1 illustrates the three critical components of MagLIF: magnetization, laser heating, and compression. The axial magnetic field inhibits radial thermal conduction loss throughout the implosion. Laser heating brings the fuel to a precompression temperature on the order of $100 \mathrm{eV}$. Simulations indicate that the initial axial and azimuthal anisotropies in the heating profile smooth out over the remaining 50-60 ns of the implosion [10]. The magnetically driven liner implosion [7] compresses and further heats the fuel to fusion-relevant conditions through PdV work.

In MagLIF, fuel areal density at stagnation is 2 orders of magnitude below the typical ICF value required for ignition; thus, the concept relies on both magnetic flux compression and liner tamping of the fuel for confinement [11]. As a result, liner stability throughout the implosion is critical for effective target performance. Years of experiments studying

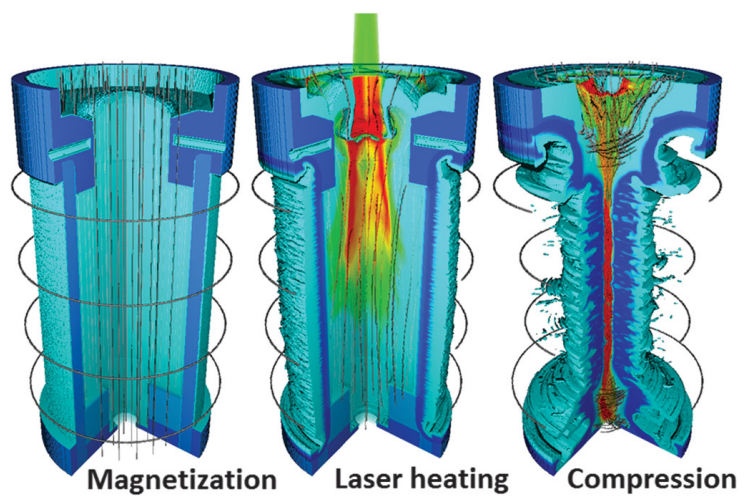

FIG. 1 (color). A schematic representation of the three critical components of the MagLIF concept. An axial current creates a $J_{z} \times B_{\Theta}$ force that is used to implode a gas-filled, premagnetized, cylindrical target. Near the start of the implosion, the fuel is heated by the laser. The liner compresses and further heats the fuel to fusion-relevant conditions at stagnation. 
liner stability, along with sufficient fidelity in related liner dynamics calculations, suggested that magneto-RayleighTaylor instability growth [12] would not preclude successful integrated experiments utilizing a liner with an aspect ratio $(\mathrm{AR}) \leq 6\left(\mathrm{AR}=R_{\text {outer }} / \Delta R_{\text {wall }}\right)$ [13-17].

In the first integrated experiments, the target was an AR 6 beryllium liner with an inner radius of $2.325 \mathrm{~mm}$. The imploding portion of the liner was $7.5 \mathrm{~mm}$ tall, with a $0.5 \mathrm{~mm}$ tall aluminum cushion [17] above and a $1 \mathrm{~mm}$ tall nylon cushion below to mitigate the previously observed wall instability [16]. The initial fuel densities used in these experiments were approximately 0.7 and $1.5 \mathrm{mg} / \mathrm{cm}^{3}$. The laser entrance hole (LEH) window was a polyimide foil with an initial thickness of $3.4 \pm 0.2 \mu \mathrm{m}$, which was deformed into approximately a spherical cap with radius $1.5 \mathrm{~mm}$ and height approximately $0.6 \mathrm{~mm}$. The LEH was located $1.5 \mathrm{~mm}$ above the imploding region of the target to avoid mixing laser-accelerated window material into the fuel [10].

The target was premagnetized to $10 \mathrm{~T}$ using the applied $B$ field on $Z$ (ABZ) system [18]. The $Z$ beamlet laser (ZBL), a $2.5 \mathrm{~kJ}, 1 \mathrm{TW}$, frequency-doubled $\mathrm{Nd}$ :YAG laser [19], heated the deuterium fuel. The $100 \mathrm{~ns}$ rise time, 19 MA current of the $Z$ machine [20,21] drove the liner, which compressed the fuel. The experimental drive current, experimental laser power, and simulated liner trajectory [10] are plotted in Fig. 2. The laser energy was temporally split into two pulses. The $500 \mathrm{~J}$ prepulse led the main pulse by $4 \mathrm{~ns}$ and was intended to disassemble the LEH window in order to increase both the transmission fraction of the main laser pulse and the associated fuel heating [10].
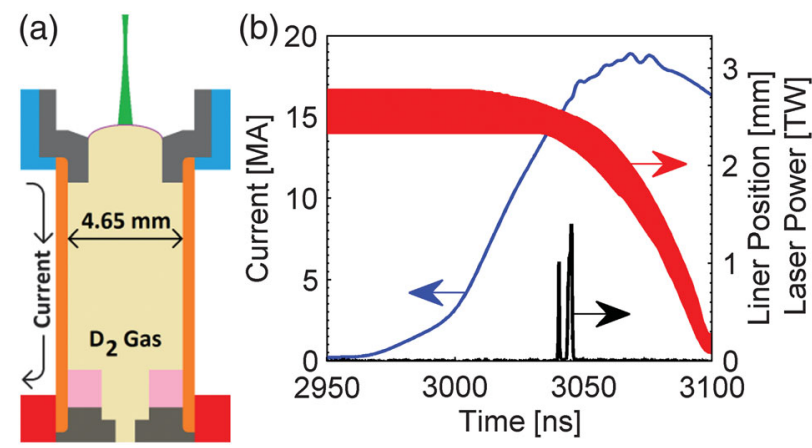

FIG. 2 (color). (a) The target geometry used in these experiments. The anode is shown in blue and the cathode is red. The Be target (orange) has an aluminum cushion (gray) at the top and a nylon cushion (pink) at the bottom. The yellow region indicates the deuterium gas fill. The LEH is shown above the target, and the gas fill tube is attached to the bottom. The approximate laser path is shown in green. The laser focus was approximately $3.5 \mathrm{~mm}$ above the LEH window, which produced a defocusing beam with a spot roughly $0.45 \mathrm{~mm}$ square at the window surface $\left(I \approx 5 \times 10^{14} \mathrm{~W} / \mathrm{cm}^{2}\right)$. (b) The nominal drive current (blue), simulated implosion trajectory [10] (red), and laser power (black) for these experiments. The uncertainty in the peak drive current is 1-2 MA and the uncertainty in the laser power is $10 \%-20 \%$.
A series of experiments were conducted in which nominally identical targets were fielded, but the use of laser heating and applied magnetic field was varied. The measured primary deuterium-deuterium (DD) and secondary deuterium-tritium (DT) neutron yields and inferred ion and electron temperatures for these experiments are given in Fig. 3. In the best performing experiment, the ion and electron temperatures at stagnation were $2.5 \pm 0.8$ and $3.1_{-0.5}^{+0.7} \mathrm{keV}$, respectively, and the DD yield was $2.0 \pm 0.4 \times 10^{12}$. In null experiments that did not incorporate both laser heating and an insulating magnetic field, the stagnation temperature was $\leq 1 \mathrm{keV}$ and the DD yield
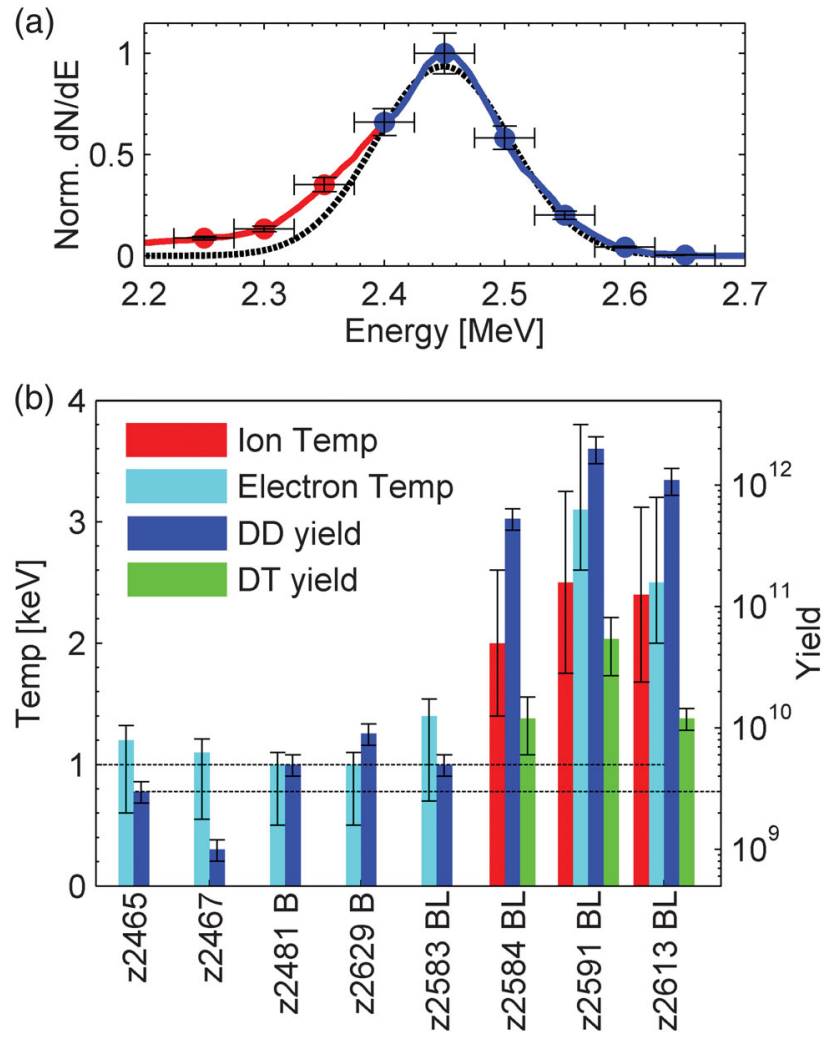

FIG. 3 (color). (a) An example NTOF spectrum from experiment z2591. The experimental data used to fit the Gaussian (dashed black line) are plotted in blue. Data on the low energy side of the peak (red) deviate from a Gaussian, which may be due to a variety of effects (e.g., neutron scattering); these points are not included in the fit. The uncertainty in the width of the energy distribution shown here is a conservative estimate based on the instrument response. (b) The electron and ion temperatures inferred from x-ray and NTOF spectra, respectively, and the DD and DT neutron yields. Experiments using a magnetic field are labeled with a B, and experiments using laser heating are labeled with an L. All experiments used approximately $0.7 \mathrm{mg} / \mathrm{cm}^{3}$ initial fuel density except for experiments z2481 and z2583, which used $1.5 \mathrm{mg} / \mathrm{cm}^{3}$. The dashed line at $1 \mathrm{keV}$ represents the approximate lower limit of the electron temperature measurement technique. The dashed line at $3 \times 10^{9}$ represents the approximate background for the DD measurement. The DT measurement floor is $7 \times 10^{7}$. 
did not exceed $10^{10}$. The background for the DD yield measurement was approximately $3 \times 10^{9}$, which was on the same order as the yield in the null experiments. Note that one fully integrated experiment (z2583) failed to produce significant yield. This experiment was nominally identical to the others but with a higher initial fuel density $\left(1.5 \mathrm{mg} / \mathrm{cm}^{3}\right)$. This result is not fully understood nor reproduced at this time. The best performing integrated experiment produced a DD yield that exceeded the best performing nonfully integrated experiment by a factor of $>200$.

The neutron diagnostic suite for these experiments consisted of indium and copper activation diagnostics as well as neutron time of flight (NTOF) detectors [22,23]. Nine indium activation samples spread over three polar angles, $20^{\circ}, 90^{\circ}$, and $170^{\circ}$, were fielded to measure the DD yield. The difference in the yield between these locations was less than the uncertainty in the measurement $(25 \%)$ indicating isotropic neutron generation. NTOF detectors were fielded at polar angles of $78^{\circ}$ and $180^{\circ}$ to examine the neutron spectrum. The ion temperature of the stagnation plasma was inferred from a Gaussian fit to the high energy side of the DD neutron peak in each spectrum; the average from multiple detectors is reported. An example fit is shown in Fig. 3. In experiments with measurable yield, average ion temperatures were in the range of 2 to $2.5 \mathrm{keV}$. Secondary DT neutrons, a result of the tritons produced in the aneutronic branch of the DD reaction [23], were measured using copper activation and the NTOF spectra. DT yields (up to $5 \times 10^{10}$ ) were measureable only in experiments with significant DD yield.

Filtered diamond photoconducting detectors (PCDs) [24], silicon diode detectors (SiDs) [24], and time-resolved $\mathrm{x}$-ray imaging [25] were fielded to record $\mathrm{x}$-ray emission from the target. Sample data from these diagnostics are presented in Fig. 4. SiDs filtered for different photon energies indicate that there were two temporal components to the radiation pulse in fully integrated experiments, first a high energy component that originated from the fusion plasma at the center of the target, and later a lower energy component due to emission from the exterior surface of the liner. The FWHM of the peak associated with the fuel stagnation was approximately $2 \mathrm{~ns}$, which is consistent with the time-resolved $x$-ray images. During the first peak in the $\mathrm{SiD}$ signal there was a narrow column in the $\mathrm{x}$-ray image, and during the second peak there was emission from the exterior of the liner. Emission from the liner exterior is observed in experiments without laser heating [26] as well as fully integrated experiments. Emission from the fuel stagnation column was observed only in experiments with both laser heating and magnetization.

High energy $(>7 \mathrm{keV})$, time-integrated, 1D spatially resolved x-ray spectra [27] were combined with absolutely calibrated PCD signals to infer additional details of the stagnation conditions. Axially resolved spectra from
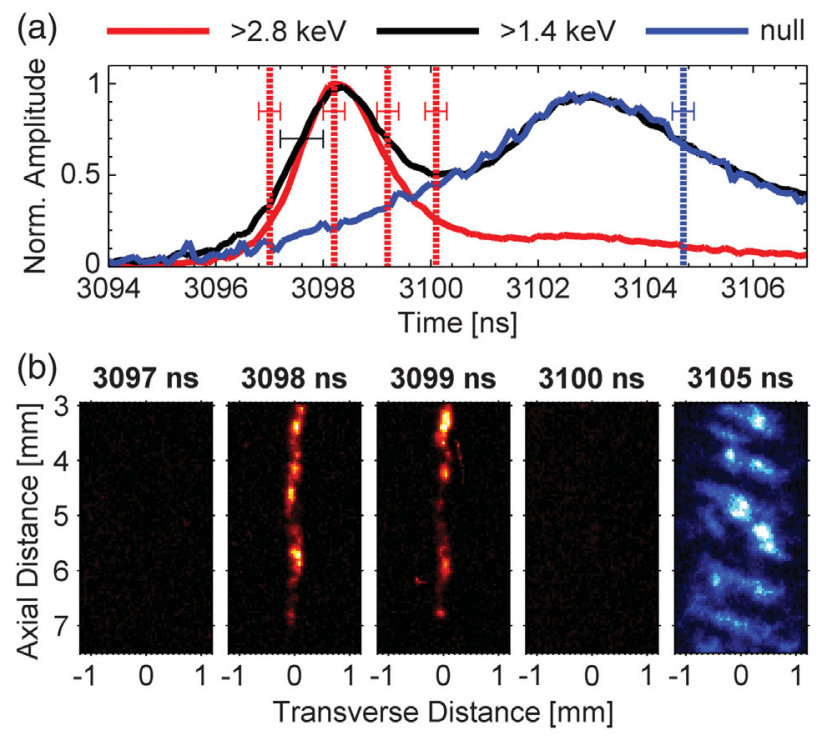

FIG. 4 (color). (a) Representative SiD signals for fully integrated experiments filtered by $100 \mu \mathrm{m}$ Kapton $(>2.8 \mathrm{keV})$ and $25 \mu \mathrm{m}$ Kapton $(>1.4 \mathrm{keV})$ and an experiment without laser heating filtered by $25 \mu \mathrm{m}$ Kapton (null). Timing uncertainties in the measurements are represented by horizontal bars. (b) X-ray emission at the five times indicated by the vertical lines in the plot. The first four images are $>2.8 \mathrm{keV}(100 \mu \mathrm{m}$ Kapton) and the final image is $>0.8 \mathrm{keV}(8 \mu \mathrm{mBe}+1 \mu \mathrm{m} \mathrm{CH})$. Fuel stagnation emission is observed at 3098 and 3099 ns; an example of liner emission is shown at $3105 \mathrm{~ns}$. In these images, the fuel emission FWHM is $170 \mu \mathrm{m}$, which is at the resolution limit of the instrument; the true emission region width is smaller, as shown in Fig. 5. In simulations, the fuel-liner interface at stagnation is approximately $125 \mu \mathrm{m}$. The upper ABZ coil limits the diagnostic field of view to the bottom $4.5 \mathrm{~mm}$ of the target.

experiments with both magnetic field and laser heating show an emission height of approximately $6 \mathrm{~mm}$ with $\mathrm{mm}-$ scale brighter regions that occupy about half of the axial extent. PCD signals indicate that the approximately $2 \mathrm{~ns}$ burst associated with fuel stagnation produces 10-20 J of x rays above $h \nu=4 \mathrm{keV}$ and $12-25 \mathrm{~J}$ above $2 \mathrm{keV}$. The ratio of these x-ray yields and the continuum slope are both consistent with the substantial attenuation of a $T_{e} \approx 3 \mathrm{keV}$ continuum source through approximately $0.9 \mathrm{~g} / \mathrm{cm}^{2}$ of cool Be liner material ( $T_{e} \approx 4 \mathrm{keV}$ if liner opacity is neglected). Observation of weak $K$-shell emission from mid- $Z$ impurities in the Be alloy indicates less than $10 \%$ atomic Be mix. Absolute x-ray yields increase with both density and liner mix [28]; the inferred temperature and the measured $\mathrm{x}$-ray emission volume, duration, and yields are consistent with a hot plasma density of $0.4 \pm 0.2 \mathrm{~g} / \mathrm{cm}^{3}$ and $<10 \%$ atomic Be mix.

A time-integrated, spherical crystal optic diagnostic was used to image x-ray emission from the full target height at stagnation. The diagnostic is similar to the bent crystal monochromatic imaging system used for radiography on $Z$ [29]. This new system differs from the radiography system 


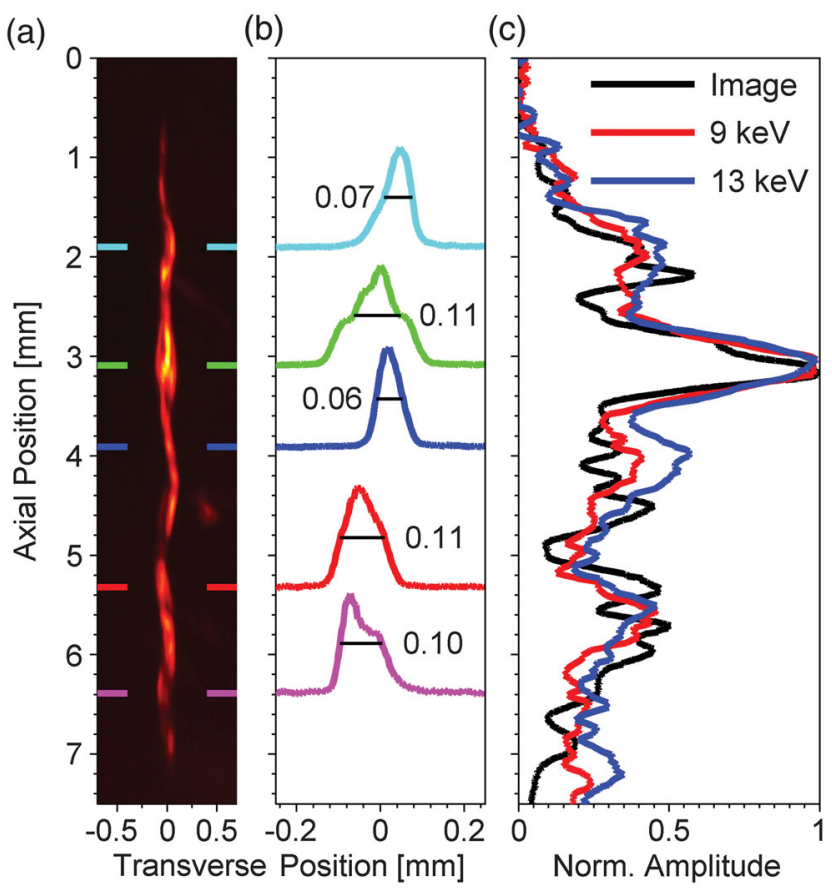

FIG. 5 (color). (a) Time-integrated self-emission image of the hottest region of the fuel at stagnation. (b) Horizontal lineouts of the stagnation image at five axial positions. The numbers next to each lineout indicate the FWHM in mm. (c) An axial lineout integrated over the full width of the stagnation image from -0.13 to $0.13 \mathrm{~mm}$ (black) and axial lineouts of the $\mathrm{x}$-ray spectrum at $9.4 \pm 0.5 \mathrm{keV}$ (red) and $13 \pm 1 \mathrm{keV}$ (blue).

in that there is no backlighting source and a $\mathrm{Ge}(220)$ crystal is used. The diagnostic is sensitive to $n \times 3.12 \mathrm{keV}$ photons where $n$ is a positive integer representing the crystal diffraction order. The axial and radial resolutions of the system are approximately 80 and $60 \mu \mathrm{m}$, respectively.

The image from experiment z2613 is shown in Fig. 5. The image shows a weakly helical stagnation column approximately $6 \mathrm{~mm}$ tall with a radial displacement of $0.05 \pm 0.02 \mathrm{~mm}$ and an axial wavelength of $1.3 \pm 0.3 \mathrm{~mm}$. The FWHM of the stagnation column cross section varies between approximately 60 and $120 \mu \mathrm{m}$ with axial location. Small levels of asymmetry, which are at the resolution limit of the diagnostic, are present in the transverse lineouts.

The liner opacity was sufficient to eliminate the $3.1 \mathrm{keV}$ contribution to the image, and based on the high energy $\mathrm{x}$-ray spectra and the energy-dependent crystal reflectivity, the signal intensity for $n=4$ and above was negligible as well. The result is an image primarily composed of 6.2 and $9.4 \mathrm{keV}$ photons. In Fig. 5 an axial lineout of the image is compared to axial lineouts of the $\mathrm{x}$-ray spectrum at $9.4 \pm 0.5$ and $13 \pm 1 \mathrm{keV}$. The origin of the axial structure in the lineouts is still under investigation, but it is likely a combination of variations in both fuel emission intensity and liner opacity.

A critical component of any MIF concept is flux compression of the seed magnetic field. In these experiments, significant magnetic flux compression is demonstrated by the ratio of the DT yield to the DD yield and by the DT neutron spectra. In nonmagnetized, spherical DD implosions, the ratio of the DT yield to the DD yield has been proposed as a diagnostic for the effective $\rho R$ of the fuel [30]. For the present experiments with hot fuel density of approximately $0.4 \mathrm{~g} / \mathrm{cm}^{3}, 50 \pm 20 \mu \mathrm{m}$ radius, around $6 \mathrm{~mm}$ height, and $T_{e} \approx 3 \mathrm{keV}$, the expected DT:DD ratio is below $10^{-3}$. The measured ratio, however, is above $10^{-2}$, indicating that the magnetic field is extremely effective at trapping the fast tritons. The measured DT:DD ratios are also consistent with relatively small mix fractions of $\operatorname{Be}(<10 \%)$ in the hot, neutron-producing fuel. A detailed explanation of this analysis for an idealized plasma column, as well as the relationship between the shape of the DT spectra and the magnetic field, are given in a companion paper [31].

The results from these experiments validate key features of the MagLIF concept. The NTOF and x-ray spectra indicate that the stagnation plasma reached fusion-relevant temperatures only when both magnetization and laser heating were utilized. This is consistent with expectations since the implosion velocity $(70 \mathrm{~km} / \mathrm{s})$ is too low to generate such high temperatures in a nonmagnetized, nonpreheated target. The isotropic, near-Gaussian DD NTOF spectra, DD yield isotropy, high ion and electron temperatures (with $T_{i} \approx T_{e}$ ), and the large secondary DT yield provide evidence for a thermonuclear origin of the yield rather than beam-target reactions, which have been suggested as a significant source of yield in other magnetically driven implosions [32-34]. The range of measured DD yields $\left(5 \times 10^{11}-2 \times 10^{12}\right)$ is encompassed by the $2 \times 10^{11}-6 \times 10^{13}$ thermonuclear yield range estimated based on the $0.2-0.6 \mathrm{~g} / \mathrm{cm}^{3}$ fuel density (from $\mathrm{x}$-ray yield), $2-3.1 \mathrm{keV}$ temperature (from NTOF and $\mathrm{x}$-ray spectra), $0.02-0.05 \mathrm{~mm}^{3}$ volume (from x-ray imaging), and $1-2 \mathrm{~ns}$ duration (from $\mathrm{x}$-ray emission history). Given these stagnation parameters, the calculated electron-ion collision time is $\ll 1 \mathrm{ps,}$ which supports the thermal equilibrium of electrons and ions observed in the x-ray and NTOF spectra.

The MagLIF concept relies on the combination of laser heating the fuel to $100 \mathrm{~s}$ of $\mathrm{eV}$ and cylindrical compression to reach multi-keV temperatures. Liner stagnation begins when the plasma pressure exceeds the drive pressure. In simulations, the radius at which stagnation occurs increases with the preimplosion temperature [6,10]. The successful target performance in these experiments is promising since the designs given in Ref. [6] utilize greater laser energy; thus, those targets are predicted to converge a factor of 1.5-2 times less [10].

While these initial experiments demonstrated thermonuclear yields, fusion-relevant stagnation temperatures, magnetic flux compression, and self-consistent results, preshot simulations predicted DD yields in excess of $10^{13}$ [10]. 
Possibilities for this discrepancy include liner-fuel mix and 3D effects, but the leading hypothesis is poor laser coupling through the LEH. The simulations [10] did not account for laser-plasma interaction losses in the window or gas, used local diffusion models, and assumed a spatially smooth laser beam profile, which is expected to transmit a greater fraction of the laser energy through the LEH than a nonsmoothed laser beam, as was used in these experiments. Yields comparable to experiments are obtained in simulations that assume transmission of approximately $5 \%-10 \%$ of the laser energy into the fuel [10]. Experiments to test laser transmission through the LEH and improvements to the laser are presently under way. Enhanced target performance due to improved laser heating may be possible in future experiments.

The authors would like to thank the $Z$ machine operations team, the $\mathrm{ZBL}$ operations team, the $\mathrm{ABZ}$ operations team, and the target fabrication team for their contributions to this work. Sandia National Laboratories is a multiprogram laboratory managed and operated by Sandia Corporation, a wholly owned subsidiary of Lockheed Martin Corporation, for the U.S. Department of Energy's National Nuclear Security Administration under Contract No. DE-AC04-94AL85000.

[1] J. Lindl, Phys. Plasmas 2, 3933 (1995).

[2] N. B. Meezan et al., Phys. Plasmas 17, 056304 (2010).

[3] L. J. Perkins, R. Betti, K. N. LaFortune, and W. H. Williams, Phys. Rev. Lett. 103, 045004 (2009).

[4] I. R. Lindemuth and R. C. Kirkpatrick, Nucl. Fusion 23, 263 (1983).

[5] I. R. Lindemuth and M. M. Widner, Phys. Fluids 24, 746 (1981).

[6] S. A. Slutz, M. C. Herrmann, R. A. Vesey, A. B. Sefkow, D. B. Sinars, D. C. Rovang, K. J. Peterson, and M.E. Cuneo, Phys. Plasmas 17, 056303 (2010).

[7] M. E. Cuneo et al., IEEE Trans. Plasma Sci. 40, 3222 (2012).

[8] P. Y. Chang, G. Fiksel, M. Hohenberger, J. P. Knauer, R. Betti, F. J. Marshall, D. D. Meyerhofer, F. H. Seguin, and R. D. Petrasso, Phys. Rev. Lett. 107, 035006 (2011).

[9] M. Hohenberger, P. Y. Chang, G. Fiksel, J. P. Knauer, R. Betti, F. J. Marshall, D. D. Meyerhofer, F. H. Seguin, and R. D. Petrasso, Phys. Plasmas 19, 056306 (2012).

[10] A. B. Sefkow, S. A. Slutz, J. M. Koning, M. M. Marinak, K. J. Peterson, D. B. Sinars, and R. A. Vesey, Phys. Plasmas 21, 072711 (2014).

[11] M. M. Basko, A. J. Kemp, and J. Meyer-ter-Vehn, Nucl. Fusion 40, 59 (2000).

[12] E. G. Harris, Phys. Fluids 5, 1057 (1962).

[13] D. B. Sinars et al., Phys. Rev. Lett. 105, 185001 (2010).

[14] D. B. Sinars et al., Phys. Plasmas 18, 056301 (2011).

[15] R. D. McBride et al., Phys. Rev. Lett. 109, 135004 (2012).
[16] R. D. McBride et al., Phys. Plasmas 20, 056309 (2013).

[17] T. J. Awe et al., Phys. Plasmas 21, 056303 (2014).

[18] D. C. Rovang et al., "Pulsed-coil magnetic systems for applying uniform 10-30 Tesla fields to cm-scale targets on Sandia's Z Facility" (to be published).

[19] P. K. Rambo et al., Appl. Opt. 44, 2421 (2005).

[20] M. E. Savage, K. R. LeChien, M. R. Lopez, B. S. Stoltzfus, W. A. Stygar, D. S. Artery, J. A. Lott, and P. A. Corcoran, in Proceedings of the 18th International Pulsed Power Conference, Chicago, Illinois, 2011 (IEEE, Chicago, Illinois, 2011), pp. 983-990.

[21] D. V. Rose, D. R. Welch, E. A. Madrid, C. L. Miller, R. E. Clark, W. A. Stygar, M. E. Savage, G. A. Rochau, J. E. Bailey, T. J. Nash, M. E. Sceiford, K. W. Struve, P. A. Corcoran, and B. A. Whitney, Phys. Rev. ST Accel. Beams 13, 010402 (2010).

[22] C. L. Ruiz, G. W. Cooper, S. A. Slutz, J. E. Bailey, G. A. Chandler, T. J. Nash, T. A. Mehlhorn, R. J. Leeper, D. Fehl, A. J. Nelson, J. Franklin, and L. Ziegler, Phys. Rev. Lett. 93, 015001 (2004).

[23] K. D. Hahn, G. W. Cooper, C. L. Ruiz, D. L. Fehl, G. A. Chandler, P. F. Knapp, R. J. Leeper, A. J. Nelson, R. M. Smelser, and J. A. Torres, Rev. Sci. Instrum. 85, 043507 (2014).

[24] M. C. Jones, D. J. Ampleford, M. E. Cuneo, R. Hohlfelder, C. A. Jennings, D. W. Johnson, B. Jones, M. R. Lopez, J. MacArthur, J. A. Mills, T. Preston, G. A. Rochau, M. Savage, D. Spencer, D. B. Sinars, and J. L. Porter, Rev. Sci. Instrum. 85, 083501 (2014).

[25] B. Jones, C. Deeney, C. A. Coverdale, C. J. Meyer, and P. D. LePell, Rev. Sci. Instrum. 77, 10E316 (2006).

[26] T. J. Awe et al., Phys. Rev. Lett. 111, 235005 (2013).

[27] D. B. Sinars, D. F. Wenger, S. A. Pikuz, B. Jones, M. Geissel, S. B. Hansen, C. A. Coverdale, D. J. Ampleford, M. E. Cuneo, L. A. McPherson, and G. A. Rochau, Rev. Sci. Instrum. 82, 063113 (2011).

[28] T. Ma et al., Phys. Rev. Lett. 111, 085004 (2013).

[29] D. B. Sinars, G. R. Bennett, D. F. Wenger, M. E. Cuneo, D. L. Hanson, J. L. Porter, R. G. Adams, P. K. Rambo, D. C. Rovang, and I. C. Smith, Rev. Sci. Instrum. 75, 3672 (2004).

[30] M. D. Cable and S. P. Hatchett, J. Appl. Phys. 62, 2233 (1987), and references therein.

[31] P. F. Schmit et al., following Letter, Phys. Rev. Lett. 113, 155004 (2014).

[32] C. A. Coverdale, C. Deeney, A. L. Velikovich, J. Davis, R. W. Clark, Y. K. Chong, J. Chittenden, S. Chantrenne, C. L. Ruiz, G. W. Cooper, A. J. Nelson, J. Franklin, P. D. LePell, J. P. Apruzese, J. Levine, and J. Banister, Phys. Plasmas 14, 056309 (2007).

[33] A. L. Velikovich, R. W. Clark, J. Davis, Y. K. Chong, C. Deeney, C. A. Coverdale, C. L. Ruiz, G. W. Cooper, A. J. Nelson, J. Franklin, and L. I. Rudakov, Phys. Plasmas 14, 022701 (2007).

[34] D. Klir, J. Kravarik, P. Kubes, K. Rezac, S. S. Ananev, Y. L. Bakshaev, P. I. Blinov, A. S. Chernenko, E. D. Kazakov, V. D. Korolev, G. I. Ustroev, L. Juha, J. Krasa, and A. Velyhan, IEEE Trans. Plasma Sci. 37, 425 (2009). 